\title{
Entretien avec Ezza Agha Malak ${ }^{1}$
}

\author{
Efstratia Oktapoda \\ Paris Sorbonne Université
}

Dans la nouvelle littérature française et francophone, Ezza Agha Malak est une figure emblématique de la littérature du postmodernisme. Directrice de recherches à l'Université libanaise, Médaille du Mérite de l'Éducation parlementaire, Chevalier de l'ordre des Arts et des Lettres depuis 2012, Officier de l'ordre des Arts et des lettres depuis 2011, Ezza Agha Malak est détentrice de plusieurs prix et trophées des institutions libanaises. Auteure de plus d'une trentaine d'ouvrages en français (romans, recueils de poèmes, essais), elle a signé une centaine d'études centrées sur des thèmes sociétaux et humanistes. Six ouvrages collectifs examinent son œuvre, réalisés par des chercheurs internationaux. Son œuvre (traduite en plusieurs langues, dont l'anglais et l'arabe) a fait l'objet de plusieurs thèses de doctorat et de mémoires de Master.

Ezza Agha Malak est née à Tripoli, au Liban, ville méditerranéenne qu'elle célèbre dans la plupart de ses écrits. Tenant à sa Méditerranéité, elle vit entre les deux villes méditerranéennes, Marseille et Tripoli, ses deux « eldorados », comme elle dit.

EO (Efstratia Oktapoda) : Ezza Agha Malak, vous êtes une écrivaine prolifique et une figure incontournable des Salons du livre de Paris, de Beyrouth, de Roumanie, de l'Espagne, du Maghreb ou même de la Chine, vous multipliez présentations et conférences partout dans le monde, surtout à Beyrouth, où vous présentez tous les ans vos livres, romans, poèmes et collectifs qui connaissent tous un grand succès, surtout vos romans que j'aime tant. Comment expliquezvous cet engouement pour la littérature et ce long parcours littéraire ?

EAM (Ezza Agha Malak) : C'est une question à tiroirs! Une idée en soulève une autre. Commençons alors par le premier tiroir, l'écrivaine prolifique comme vous me qualifiez. En effet, je fais paraitre chaque année un ouvrage, parfois même deux, que je signe au Salon du Livre de Beyrouth d'abord, ensuite dans d'autres Salons. Mais le plus important pour moi, c'est le Salon de Beyrouth parce que c'est chez moi ! C'est trop, dites-vous, comment peut-on arriver à écrire tout cela ?

Je dois dire que je travaille une dizaine d'heures par jour, sans me lasser. L'écriture a toujours été pour moi un refuge et un abri. J'y ai recours dans

${ }^{1}$ Entretien effectué par Efstratia Oktapoda le 2 mai 2017 à Paris.




ma joie comme dans ma détresse. Elle est parfaitement thérapeutique. Voilà un autre tiroir : l'écriture, qui a en fait deux visages : l'un est bénéfique et avenant, en un mot, bon, l'autre maléfique et malfaisant, bref malin. Cela nous pousse à nous demander si le roman méchant n'existe pas !

Alors l'écriture est mon soutien et mon réconfort. Elle vient à mon secours lorsqu'on me fait mal. Avec mes mots, j'attaque. Je l'ai toujours dit : «Mes mots sont ma force et mon arme ». Ils ont un pouvoir magique, les mots. Ils peuvent se transformer en projectiles. Avec ces outils graphiques, tu élèves ou rabaisses, tu aimes ou détestes. Certes, il vaut mieux utiliser ces outils de communication à des fins bénéfiques, comme l'amour, l'amitié, la fraternité et l'humanité, ce que je fais souvent, mais parfois il arrive que l'on se trouve contraint de lever cette arme contre ceux qui nous trahissent, qui nous détestent, qui nous font mal, et là... on tire. Mais il faut savoir bien tirer d'abord, bien viser, n'est-ce pas ? Moi je sais bien viser. J'aime beaucoup cette expression que j'ai lue quelque part : " Les mots ressemblent à des clefs. Bien choisis et intelligemment utilisés, avec sagacité, ils sont capables de fermer une bouche ou ouvrir un cœur!» Et ça m’est arrivé. Avec mes mots bien choisis, j’ai ouvert des cœurs, mais aussi j’ai fermé des bouches !!

EO : Ce qui attire l'attention dans votre œuvre, c'est l'évocation permanente de la Méditerranée. Qu'est-ce qui vous amène à l'adopter dans presque toute votre œuvre, tant romanesque que poétique?

EAM : La Méditerranée ? J'ai avec elle une longue histoire de voisinage et d'amour. "La Méditerranée ! Mon histoire avec elle est très longue. Elle date d'un demi-siècle. Elle est interminable, mon histoire avec elle », dit Monsieur Raymond dans La Dernière des Croisés. Pour ce personnage (comme pour moi), "la Méditerranée s'étend incommensurablement devant lui ».

Alors... Passons à un autre tiroir !

Ma Méditerranée n'est pas que l'espace aquatique que limite l'horizon que j'aperçois de chez moi, à Tripoli ou à Marseille, ce n'est pas non plus la mer bleue aux rivages variés et qui s'oppose au ciel, la Méditerranée est cet espace mythologique et légendaire avant toute autre chose. Quand je revois, par mon esprit, la Méditerranée, Al Bahr El-Abiad Al-Moutawassett (masculin en arabe, et c'est significatif), c'est une multitude de figures mythiques et mythologiques qui en sortent, qui se dressent devant moi en balisant la longue Histoire de mon pays, que je trouve passionnante. C'est Zeus qui a enlevé Europe, la princesse phénicienne pendant qu'elle jouait dans les jardins de son père Agénor, le roi de Tyr ; Zeus qui a chevauché la mer en l'emportant sur son dos ; qui a fondé avec elle un continent qui porte aujourd'hui le nom d'une princesse phénicienne, «Europe ». C'est le 
roi Melqart qui a découvert le murex grâce à son chien (qui revient de sa trotte, la gueule maculée de pourpre) ; c'est Ahiram qui a envoyé au roi Salomon, le bois du cèdre, avec des maçons et des architectes pour l'aider à construire le temple de Jérusalem ; c'est Cadmos, allé à la recherche de sa sœur Europe, sur l'ordre de son père et, désespéré de la trouver, n'osant plus retourner en Phénicie sans elle, qui avait fondé Carthage. Quand je repense la Méditerranée, je revois les Phéniciens qui l'ont traversée pour installer leurs comptoirs et répandre leur alphabet, le prototype de tous les alphabets actuels ; qui vendaient leurs marchandises de haute qualité dans les villes méditerranéennes sans pour autant faire aucune guerre ; c'est leurs colonies pacifiques qui, en construisant Carthage, ont entrepris des liens de fraternité et d'amitié dans le pourtour méditerranéen ; c'est aussi Elissar ou Didon, la reine de Carthage, et son frère Pygmalion. C'est Adonis le dieu du renouveau et de la renaissance dont le culte se retrouvera plus tard dans la mythologie grecque, tout comme Astarté, assimilée à l'Aphrodite des Grecs. La Méditerranée c'est toutes ces légendes mêlées... Il y en a d'autres encore et encore! Quand je dis Méditerranée, je vois la Phénicie... Elles sont liées... J'ai longtemps appris que l'histoire du Liban est écrite non seulement sur fond phénicien, mais surtout sur fond de Méditerranée qu'avaient traversée les Phéniciens. Il y avait quelque chose de purement romanesque notamment à l'égard de ces sociétés venues d'une autre époque et qui figurent comme des acteurs de la grande Histoire. Pour moi, c'est une Méditerranée nourrie de mythes et de légendes.

\section{EO : Comment pouvez-vous résumer ce recours littéraire quant à la forte présence de la Méditerranée dans votre œuvre?}

EAM : La Méditerranée comme je la représente dans mon œuvre, fonctionne doublement : comme un élément poétique et comme un élément romanesque. Mais d'autres dimensions viennent s'y ajouter. Certes, il y a le côté historique actuel, l'humain, je veux dire. Je dois d'abord préciser que la Méditerranée, c'est mon enfance d'hier et mon refuge d'aujourd'hui. Chaque année, je lui consacre deux ou trois mois de l'été pour y plonger tous les jours. Si je retourne en été au Liban, c'est pour la visiter, et longuement; pour « plonger dans son sein frais » comme je le dis dans un de mes poèmes. Tu vois, elle est pour moi symbole et mythe, passé et présent. Comment ne pas en faire l'acteur principal et le personnage premier de mes romans et de ma poésie?

Comme vous le dites, l'image de la Méditerranée accompagne presque tous mes écrits, romans, poésie, conférences, etc. Est-ce conscient ou inconscient, ce recours ? Je ne le sais pas. Mais parfois je le fais exprès. Je situe un événement au bord de la Méditerranée, ou dedans. Je ne peux pas la passer sous silence et il y a de quoi. Je suis fière d'appartenir à cette région méditerranéenne riche d'histoire. Ma méditerranéité compte beaucoup pour moi. 


\section{EO : Vous évoquez aussi les villes méditerranéennes et vous y situez} les événements. Est-ce pour servir la cause romanesque?

EAM : La cause romanesque a certes, besoin d'un cadre. Souvent, ce cadre est constitué par les villes de mon pays d'abord. Je viens d'un pays profondément méditerranéen qui, le long d'un littoral aux cordons variés, s'étend sur $250 \mathrm{~km}$ de long (seulement) pour $50 \mathrm{~km}$ de largeur (seulement) en se rétrécissant vers le sud. Cette forme géographique m'emballe toujours. Les villes les plus importantes se situent sur la côte. Tripoli, Jbeil (ou Byblos), Beyrouth, Sidon, Tyr. Ce sont des villes phéniciennes célèbres pour leurs châteaux et leurs ports d'où partaient les Phéniciens avec leurs vaisseaux chargés de marchandises. Des villes que j’aime, en effet, évoquer avec délectation dans mes écrits, même quand elles ne font pas partie urgente du paysage narratif. Dans mon dernier roman La Paix, l'Amour et rien d'autre, je les énumère non seulement pour le besoin de la cause romanesque, mais aussi parce que la beauté de ces villes et leur histoire glorieuse à l'époque phénicienne ne me laissent pas indifférente. Et c'est ainsi que le deuxième chapitre de ce roman est intitulé «Longer la bonne mer », c'est-à-dire la Méditerranée. Au Liban, pour aller de Tripoli à Beyrouth, on roule sur une route qui borde (et que borde) la mer Méditerranée en traversant toutes ces villes côtières, qui avaient leur prestige historique. Mais ce n'est seulement pas les villes libanaises qui me servent de cadre romanesque aux événements. Les villes méditerranéennes françaises aussi. Marseille où je réside est évoquée souvent, comme ville et comme port, avec ses îles et ses châteaux de la mer. Que ce soit dans L'Homme de tous les silences ou dans La Paix, l'amour et rien d'autre, Marseille et sa Méditerranée constituent un cadre à beaucoup d'événements narratifs. Je dois dire que les villes méditerranéennes de la côte constituent instinctivement - une composante de mes histoires. Aujourd'hui, quand il s'agit de raconter la mer, deux élans opposés dirigent mon écriture, selon le sujet traité. Le premier élan est amour, adoration et passion; le deuxième, c'est la méfiance et un certain ressentiment quand il s'agit du contenu des villes, c'est-à-dire leurs habitants et leurs gouverneurs. Ce sont ces gouverneurs qui rendent les villes laides et la Méditerranée corrompue comme eux.

EO : L'histoire de la Phénicie, comme l'histoire de la Méditerranée, traverse vos romans et même votre poésie. Comment l'expliquez vous?

EAM : En effet, les Phéniciens, je les représente comme et quand je le peux. Ces aventureux Sidoniens et Tyrrhéniens, qui ont sillonné la Méditerranée, méritent l'admiration. Ils étaient un peuple pacifique qui privilégiait le dialogue et évitait de faire des guerres, en prônant la paix. 
Comme nous aurions besoin aujourd'hui de telles conceptions! C'est aussi un peuple actif et travailleur. En sillonnant la Méditerranée, ils fondèrent des comptoirs et des marchés permanents sur les deux rives, et ont fait naitre ainsi une transculturalité particulière. Leur commerce maritime se doublait d'un apport culturel. Et c'étaient les Carthaginois, colonies phéniciennes, qui avaient participé aux phénomènes culturels et guerriers. Un tel peuple est à respecter avant tout.

\section{EO : Même les ports méditerranéens ont une part d'histoire romanesque. Ce n'est pas un hasard, je suppose.}

EAM : Les ports, tous les ports, ont un positionnement géographique et historique important. Une porte d'entrée et de sortie, un point de croisement de routes maritimes. Dans une zone portuaire, il se passe toujours quelque chose. Il serait donc intéressant d'en parler dans un roman ou même de les chanter dans un poème. C'est ainsi du port de ma ville natale à l'ouest de Tripoli. Il s'appelle el-Mina, c'est-à-dire le port tout simplement. C'est un large port commercial qui fut, le 22 juin 1893, le théâtre d'une tragédie britannique dont je me suis inspirée pour écrire Tripoli, cimetière des Anglais. Le HMS Victoria entre en collision avec le Camperdown, les deux navires les plus importants de la flotte britannique envoyée dans cette partie de la Méditerranée pour (soi-disant) faire des manœuvres dans des eaux peu profondes. C'était en fait une raison apparente et un prétexte, la raison réelle c'était que la Grande-Bretagne voulait contrôler la route de la soie et créer des liens avec la Sublime porte. Le HMS Victoria a coulé bizarrement en emportant 356 membres de l'équipage, y compris le commandant de la flotte, Tryon. Ce fut sa manœuvre fatale. La Méditerranée orientale que longe Tripoli fut le premier témoin de cette catastrophe qui a secoué la Grande-Bretagne. C'est un fait historique important que j'ai reporté, avec très peu de fiction.

Je dois préciser que Tripoli, qui fut une des premières échelles du Levant, et qui s'étend le long du littoral, forme avec son port une presqu'île où sont dispersées 6 autres îles. Ce coin de la Méditerranée, je le décris dans plus d'un roman, parce qu'il constitue (pour moi) un élément romanesque intéressant.

EO : Vous avez dû faire beaucoup de recherches pour écrire ce fait historique très peu connu.

EAM : Des recherches, j'en ai fait certes, autrement comment rapporter des faits historiques qui doivent s'appuyer sur la réalité et avoir leur crédibilité et leur vérité historique ? Comme je vous l'ai dit, je travaille énormément pour la rédaction d'un roman et je ne recule devant aucun événement qui me paraît peu accessible. Il y a des moments historiques 
presque méconnus. Je m'investis alors dans la recherche des faits. C'est un travail responsable parce qu'il faut prendre en compte la vérité historique.

EO : Racontez-nous un peu Ezza, quels autres ports exploitez-vous comme élément romanesque historique, à part évidemment le port de votre ville natale?

EAM : D'autres ports sont évoqués dans d'autres romans et plusieurs régions maritimes sont aussi mentionnées. Dans La Dernière des Croisés par exemple, le port de Beyrouth est décrit presque en détail : l'embarquement et le débarquement des navires avec leur chargement, les commerçants, les matelots, les dockers, les douaniers... et tout un monde fou qui « travaille comme des fourmis ». Même le port de Gibraltar est décrit lors du passage de la flotte britannique. Je trouve beaucoup de plaisir à situer les événements dans un contexte historique.

EO : Dans La Mallette, on trouve que le sens de l'odorat participe de la présence de la Méditerranée. On peut lire à la page 84 : "Une brise humide apporta à son odorat un goût salin, fortement iodé. Qui lui dénoua en quelque sorte ses muscles tendus ». Et quelques pages plus loin, vous parlez d'" odeurs iodées " de la mer. Le sens de l'odorat devient-il un élément sensoriel indissociable de la mer? Comment l'expliquez-vous?

EAM : En fait, j'assimile l'odorat à plein d'éléments, c'est probablement parce que le sens de l'odorat est très développé chez moi et que par suite, je " sens » la mer. Alors, j’exploite cet état préventif pour l'intégrer à mes événements romanesques. La fin du roman La Dernière des Croisés, par exemple, se clôt sur un paysage méditerranéen où " l'air est salin et qui rappelle l'amant de la plage tripolitaine ». Un fonctionnement synesthésique s'en dégage. Dans Balafres on Le silence de la Méditerranée la mer a une odeur qui fait que les kidnappés aux yeux bandés réalisent, à travers leur sens olfactif, que leur tortionnaire les conduisait vers la mer, pour les abattre. Ce fonctionnement sensoriel se traduit en émotions dans l'écriture romanesque.

EO : Qu'est-ce qui fait que la Méditerranée revient fréquemment dans votre œuvre jusqu'à jouer parfois un rôle de personnage?

EAM : La Méditerranée a exercé en fait sur moi une double fascination : réelle et littéraire. Je crois que mes souvenirs d'enfance sont à l'origine de cette fascination. Si la Méditerranée est fortement présente dans presque tous mes écrits, ce microcosme aquatique revient dans mon œuvre comme la nostalgie d'un paradis perdu, comme un besoin vital où il me plaît de m'y replonger. 
Une plongée littéraire très peu différente de la plongée réelle, avec des personnages inscrits dans un monde maritime où la mer devient un lieu de mémoire. Je le fais parfois exprès, par chauvinisme peut-être, de parler de cette mer nourricière qui m'a vue grandir, que j'ai vue dans tous ses états. Dans chacun de mes écrits, je ne peux m'empêcher de célébrer sa beauté, ses rives, ses villes, ses paysages, liée pour ainsi dire, à une vision du monde maternel et à un âge d'insouciance et de jouvence.

\section{EO : Vous avez dit quelque part que les thèmes développés sont tous inspirés de la réalité. Et la part de fiction?}

EAM : Dans la conscience romanesque, tout resurgit d'un passé proche ou lointain, métaphorisé ou concrétisé. Toute œuvre est influencée par le passé de son auteur. Lorsqu'on grandit sur des rivages aimés, lorsque la Méditerranée forme une partie de notre passé, comment pourrait-on l'ignorer?

Notre passé constitue en fait notre identité. Nous nous construisons sur ses souvenirs restés gravés dans la mémoire bien plus que nos souvenirs d'adulte. Ils attendent un petit coup pour qu'ils se réveillent et sortent.

L'amour de la mer s'est développé chez moi depuis toujours. C'était mon père qui a nourri chez nous, ses enfants, cet amour, cette passion plutôt. Sur une plage presque sauvage, où il n'y avait pas encore de stations balnéaires de luxe comme aujourd'hui, mais des cabanes en bois, notre père nous emmenait presque tous les week-ends, considérant que le soleil et le vent de la mer empêchent de recourir aux toubibs. Ma mère restait à la maison parce qu'il était interdit aux femmes d'aller à la plage. Pour moi enfant, la mer était une source de jeux et de créativité. Savoir chevaucher les petites vagues, aller dans leur fond, les remonter... J'ai grandi avec ces tentatives ludiques et qui m'emmenaient un peu plus loin, vers la poésie. Les bleus de la mer et du ciel, l'horizon qui, comme une interface ou une jonction, réunit et sépare ces deux éternités, tout cela constituait pour moi un réservoir d'images. Mon père nous disait que la mer constitue une nourriture pour la vue. " Envoyez vos regards vers l'horizon, ça vous fera du bien, cela rendra le regard plus perçant, et la vue bien limée », nous exhortait-il. Il achetait des poissons de certains pêcheurs qui sortaient leurs filets pleins de poissons, ces petits habitants d'une mer avant tout « nourricière ». J'y voyais le cosmos lié à l'anthropos. L'homme et la mer. Le rapport est fort. J'y ai toujours cru. Mes écrits obéissent à cette formule. C'est l'image d'une mer nourricière que j'ai d'abord retenue. Puis celle d'une mer-mère qui a accompagné tous mes âges. C'est pourquoi elle est restée un souvenir narratif et un moment privilégié de mes réflexions intimes. 
EO : Alors c'était cela qui vous avait initiée assez tôt à l'amour de la mer, à la poésie et à l'écriture ? N'avez-vous pas retenu de cette Méditerranée que son aspect ludique et nourricier?

EAM : Dans l'écriture de la mémoire, la mer se confond avec la figure de la mère. J'ai célébré cet aspect dans plusieurs de mes poèmes. Pour moi, c'était une «mère Méditerranée ». «Ma mer-mère qui guérit les blessures » avais-je écrit dans "Amour aquatique », un poème du recueil Migration. Et même dans mes romans, c'est un peu cela. C'est à se demander s'il n'y aurait pas une écriture autobiographique. Certes, ce côté réapparaitt inexorablement. J'ai su par la suite que cette mer n'a pas toujours la surface plane et calme comme un miroir et qu'il lui arrive parfois de se déchaîner, pour des raisons cosmiques. Je dois dire que ce n'est pas seulement l'aspect amène et nourricier de la mer que mon imagination a retenu, mais aussi son aspect méchant et dangereux. C'est le jour où on a sorti de l'eau un enfant qui avait presque mon âge et qui avait péri suite à sa noyade. C'est alors que j'ai compris qu'il ne faut pas se fier aux apparences et aux surfaces calmes et qu'il fallait aller au fond des choses. La Méditerranée n'est pas que volupté. Il lui arrive de commettre des délits, parfois en raison de la bêtise humaine. La jeune fille de La Dernière des Croisés l'a bien compris, mais un peu tard.

EO : Pouvez-vous nous dire quel genre de rapports vous entretenez avec la Méditerranée?

EAM : Mon rapport avec ma Méditerranée, que j'aperçois les jours d'hiver à travers ma fenêtre, à Tripoli, et de ma terrasse à Marseille, est resté positif même quand j'ai à raconter des événements négatifs. L'évocation des paysages méditerranéens a abouti à une poétique des lieux marqués affectivement. À treize ans, j'avais couché ces sentiments et ces émotions sur du papier et plus tard sur l'écran de mon ordinateur dans des nouvelles et des romans. Ma première nouvelle «sans rendez-vous préalable » qui fut diffusé en 3 épisodes sur "Radio Liban" dans les années soixante, raconte une Méditerranée antique et mythique, et je crois que c'est grâce à mes premières impressions que j'ai eues d'elle. Cependant, penser à la guerre du Liban, longue de 20 ans et dont on subit encore les conséquences, a modifié en moi les premières impressions. Ce n'est plus la mer-mère, ma Méditerranée, mais un élément naturel cruel, une mégère restée indifférente devant nos catastrophes. Elle a perdu de son prestige suite à la guerre que le Liban a subie lors de l'occupation syrienne ; une guerre provoquée et déclenchée par la rapacité des grands États ; ces requins qui cherchent à avaler les petits États, à leur faire la guerre pour les anéantir et sucer leur sang, tel qu'on le voit aujourd'hui. C'est à ce sujet que mon roman Balafres ou Le silence de la Méditerranée fait allusion. Il décrit la guerre et la trahison d'une Méditerranée qui n'a pas su accourir à sauver un pays en danger. Au 
Liban, on inventait les combats et les guéguerres alors que le pourtour méditerranéen regardait amusé comme on regarde une pièce de théâtre. Le silence peut être coupable. Oui, c'est une mer amène, mais aussi dangereuse et cruelle.

Tout cela a influencé mon appartenance méditerranéenne, ma méditerranéité, comme vous l'avez bien définie dans l'une de vos études, comme mon parcours d'écrivain : présence anthropomorphique de la mer sous aspects métaphoriques divers, fascination et obsession, mais aussi horreur et trahison.

EO : Ezza, vous excellez dans l'écriture d'actualités intéressantes que vous situez dans un contexte historique. Doit-on comprendre que vous êtes passionnée de faits historiques quand vous racontez votre légende personnelle?

EAM : Lorsque je commence un roman, et tout en appelant aux actualités de mon présent, je convoque mes souvenirs d'enfance. Je choisis les plus émotifs, les plus ancrés dans ma mémoire pour y chercher une forme cohérente de narration et qui porte un message à ma conscience profonde. Certains événements sont propres au surgissement des souvenirs. À travers ceux-ci, nous forgeons une partie de notre personnalité et de notre rapport au monde.

C'est magnifique de vivre son histoire personnelle avec ses souvenirs d'enfance. Ces souvenirs qui se réveillent quand j'écris, deviennent grâce à ma mémoire, le matériau de mon travail. Flottant à la surface de ma conscience, ils deviennent des moments de grande netteté. Les souvenirs sont une matière à création et un moment de retour sur soi. L'écrivain peut les travailler, les exploiter et en extraire une matière à façonner. Et comme chacun se confronte différemment à ses souvenirs d'enfance en entretenant avec eux tel ou tel rapport, je peux dire que la Méditerranée a forgé ma légende personnelle, propice probablement à mon signe aquatique : le signe du cancer.

EO : Avez-vous remarqué que la représentation de la Méditerranée dans votre œuvre se fait sur un fond de réalité historique et psychologique?

EAM : C'est pendant l'écriture de La Dernière des Croisés que je découvre, à travers mes notes de recherche, que la mer est, pour l'écrivaine que je suis, un élément narratif obsessif qui revient dans tous les autres romans comme une écriture de l'inconscient. Bref, je me suis rendu compte que j'étais hantée par cette mer qui m'a vue naittre. Découverte qui m'a en quelque sorte prise sur le fait. J'étais frappée par le rôle itératif que cette mer joue dans mon écriture aussi bien poétique que romanesque. Dans ce roman, la 
mer et sa plage appellent des moments privilégiés où se nouent et se dénouent souvenirs et actualités. Dans L'Homme de tous les silences, la mer est un témoin infaillible du bonheur et du malheur de l'homme. Tout comme dans La paix, l'amour et rien d'autre.

En me lançant dans l'écriture, je tiens à ce que mon récit soit ancré dans le réel, faisant référence à des personnages et des lieux non complètement fictifs. Certes, il y aura une bonne part d'imagination, de fiction, celle qui enchaîne les épisodes d'une manière logique ou qui invente une scène vraisemblable en quelque sorte.

EO : Ezza, j'ai l'impression que ce n'est pas le même élan qui vous motive quand vous écrivez un roman ou un recueil de poèmes, ditesmoi si je me trompe.

EAM : Vous avez vu juste. En poésie, on ne cherche pas la crédibilité ni la vérité historique, comme pour le roman. La poésie suggère par petites touches et peut devenir un "anti-roman». Il y a une différence, et même une contradiction, entre le poétique et le romanesque. En poésie, la Méditerranée est orgasmique, mère, femme avec une perception charnelle, etc. Ce qui est un peu difficile de la décrire comme telle en roman. Dans les romans, elle n'a pas cette consistance inoffensive ni cette humanité universelle, mais ambiguë, que révèlent les poèmes. Lorsque mon chauvinisme méditerranéen me revient, c'est tout un élan qui me pousse à inventer et à raconter.

EO : Comment pouvez-vous expliquer son fonctionnement en tant qu'élément narratif et poétique, dans les romans d'une part et dans les poèmes d'autre part? Est-elle toujours maternelle?

EAM : La Méditerranée a vu passer les siècles et les nations et cache dans ses profondeurs les secrets de l'humanité. Source de fascination, elle est témoin des plus belles histoires d'amour. Source de plaisir et de bonheur, elle devient cependant source de malheurs et de catastrophes. Elle est maternelle, mais hostile et indifférente; bonne mère et mégère à la fois. L'aspect bénéfique dans les poèmes s'oppose à un aspect maléfique dans certains romans : opposition entre la surface et le fond, entre l'être et le paraître. Elle s'oppose à elle-même comme en oxymore. Elle n'est seulement pas témoin de scandales et de catastrophes, mais parfois elle en est à l'origine.

Le roman La Paix, l'amour et rien d'autre aborde le problème d'un petit syrien de 3 ans, qui " git inerte sur une plage sablonneuse, la tête envahie par une vague écumante en reflux». La mer, qui n'est autre que la Méditerranée, ne joue plus ici son rôle d'élément bénéfique ni poétique.

Dans La Dernière des Croisés, l'héroïne qui travaille en tant que bonne chez les riches de la côte, tombe enceinte suite à une promenade en mer 
sur le canoë de Karim, le fils du maittre. Elle a pensé, suite à ce scandale, mettre fin à ses jours en pensant au rocher appelé "rocher du suicide" planté au sein de la mer devant Beyrouth.

Dans Les Portes de la Nuit, roman construit sur le rêve et l'onirisme, Myriam revient toujours à cette mer et précisément dans un petit port au fanal élevé sur un phare tournant, pour se retrouver à côté de son amant, alors qu'elle est une femme mariée. Mis à part l'aspect psychanalytique des songes, de la rencontre et de l'expérience universelle du rêve, ce qui importe ici, c'est cette traversée de la mer (la Méditerranée) qui aboutit au désert, là où couve un grand amour, fût-il onirique.

Les écrits sont en fait la révélation de ce qu'on a longtemps caché et détenu. André Gide dit qu'un roman est «comme un archet, la caisse du violon qui rend les sons, c'est l'âme du lecteur ». Moi je dis que c'est plutôt l'âme de l'auteur, créateur et compositeur. Dans les romans, l'aspect féminin et bénéfique s'estompe parfois. La mer devient versatile, fluctuant entre mère et mégère, comme dans La Dernière des Croisés: berceuse et créatrice d'une idylle au début du roman, elle devient à la fin tentatrice et génératrice d'un scandale à répercussions graves. De même, elle est lieu de danger et de honte dans Tripoli, cimetière des Anglais; témoin de grandeur, mais aussi de décadence pour les Britanniques.

Ce n'est pas tout à fait l'Océano Nox de Hugo qui engloutit marins et capitaines "dans une mer sans fond par une nuit sans lune », mais c'est un peu comme. Dans ce roman historique, la Méditerranée qui s'étend devant Tripoli, engloutit d'une manière insolite, dans son abîme pas très profond pourtant, navire, matelots et capitaines. Un naufrage unique qui s'est produit non à cause d'un déchaînement quelconque des éléments, en cet endroit (la Méditerranée était toujours calme et déridée), c'était par outrecuidance et faute de modestie d'un amiral. L'arrogance humaine est souvent à l'origine de bien de malheurs humains.

EO : Ezza, la mer exerce une grande fascination sur votre esprit et votre pensée. C'est aussi une source d'inspiration. Vous vous inspirez toujours de ses eaux limpides où a baigné toute votre enfance. La Méditerranée anime tous vos récits. Elle est pour vous un élément romanesque de taille qui fait appel à votre sensibilité féminine d'écrivaine. Pouvez-vous nous expliquer comment cet élément naturel fonctionne comme élément narratif?

EAM : L'aspect historique de la Méditerranée est fortement présent. Si elle est, dans certains romans, le cadre amène et ludique qui passionne le narrateur, dans d'autres, elle cessera de l'être. La Méditerranée ne sera plus la mère qui "guérit les blessures et les souillures " comme je le dis en poésie. Car il lui arrive de devenir brutale et de participer aux crimes de l'humanité, comme dans Tripoli cimetière des Anglais et dans Balafres on Le silence de la Méditerranée. Là, son aspect de mégère réapparait. Alors que les 
Libanais s'entretuent, obnubilés par l'occupant qui a suivi le précepte « diviser pour mieux régner», le pourtour méditerranéen gardait un silence criminel. Même dans La Paix l'amour et rien d'autre, cet aspect virulent, voire inhumain participe de l'historicité de la narration. Dans ce roman, la Méditerranée engloutit non seulement les immigrés qui fuient à bord d'un canot, mais rejette sur la plage un bébé de trois ans, assurant ainsi aux événements, un cadre macabre.

EO : La Méditerranée a en effet plusieurs dimensions dans votre œuvre. Elle est tour à tour protectrice et dangereuse, amie et ennemie. Elle procure du bonheur, comme dans La Paix, l'amour et rien d'autre, mais elle est source de douleur, notamment dans Balafres. La Méditerranée, c'est le voyage, le salut, le chalet, l'amour. La Méditerranée représente beaucoup de choses pour vous.

EAM : En fait, cette mer n'a pas qu'une seule dimension. Elle en acquiert de nouvelles selon les circonstances de la narration et d'après les moments et les gens qui la prennent. Cessant d'être la mère qui enveloppe et protège, elle devient cet élément naturel puissant qui accumule dans son sein tous les conflits, tous les malheurs du monde. La Méditerranée est un élément pacifique lorsqu'elle s'oppose à l'océan. Élément cosmique, elle recommence toujours. Dans la deuxième partie de Tripoli cimetière des Anglais, on quitte l'océan et ses profondeurs pour entrer dans un cadre aquatique plus amène, moins agité, la Méditerranée séparée de l'océan par Gibraltar.

Élément narratif important dans mes écrits, cette Méditerranée mythique sert de cadre à des expériences psychologiques significatives. L'obstination et l'arrogance d'un amiral qui mène au naufrage; le plaisir et la volupté qu'elle procure à la jeune fille qui revient toujours à la mer, dans La Dernière des Croisés (au début de l'histoire et à sa fin). Par ailleurs, elle évoque non seulement des souvenirs historiques, mais tout un patrimoine : les plages, les îles, les souks puis les différents ports. Dans la production de la scène du naufrage, la Méditerranée devient le théâtre d'une scène réelle qui se transforme en emblème interprétable. L'angle selon lequel le navire a coulé, en perpendiculaire, représente une position insolite dans l'histoire des naufrages en temps de paix. La Grande-Bretagne aurait choisi ce coin de la Méditerranée pour, soi-disant, son peu de profondeur propice aux manœuvres de torpilles que l'amiral Tryon avait projetées.

Ici, les deux plans, psychologique et descriptif, se superposent et se juxtaposent tour à tour pour faire de la Méditerranée un arrière-fond unique à cet événement historique.

Dans L'Homme de tous les silences, la narratrice, voulant quitter le foyer conjugal, se trouve interdite de le faire en raison de son attachement à la mer qu'elle voit tous les jours. 
Dans ce roman, la narratrice est à l'image de sa créatrice, l'auteure, passionnée de Méditerranée. Et si celle-ci fonctionne doublement, à Tripoli et à Marseille, avec le même impact, ce fonctionnement persiste dans la vie réelle : c'est plusieurs fois par an que la narratrice survole la mer pour aller de l'une à l'autre de ces villes méditerranéennes: Marseille/Tripoli allerretour. Comme dans la réalité.

Dans Mariée à Paris... Répudiée à Beyrouth, c'est la même mer vue à travers la fenêtre de sa maison de Tripoli, sa ville natale et qui «déferlait nonchalamment ses vagues indolentes » comme je le dis dans le roman.

Je la fais parfois fonctionner en binôme ou même en géminée: la Méditerranée se dédouble d'une montagne, d'une vallée, d'un pré, d'un océan ou d'un désert. (...) ce dédoublement ajoute une touche agréablement pittoresque.

\section{EO : Et la Méditerranée d'aujourd'hui dans votre œuvre?}

EAM : Malheureusement, notre Méditerranée est stigmatisée de nos jours comme espace de barbarie et de repli identitaire. Elle n'est plus ce "purgatoire où se lavent les souillures", ou celle qui "déferlait nonchalamment ses vagues indolentes », au contraire, c'est la violence et le danger qui prennent le pied et c'est la phobie de la vérité qui nous prend: une culture de la violence particulièrement méditerranéenne se développe.

Dans l'espoir que la barbarie humaine s'arrête et que la brute en l'homme revienne à son humanité, nous n'aurons qu'à attendre que les grands États cessent de croquer les petits et provoquer leur destruction. Nous avons un pourtour méditerranéen qui abrite un espace digne de respect et d'admiration, où des ethnies et des religions coexistent presque pacifiquement. Je ne cesse de rappeler dans mes écrits la situation de mon pays, le Liban, et son système politico-confessionnel qui répartit les pouvoirs entre maronites, sunnites, chiites, Grecs orthodoxes, Grecs catholiques et druzes, etc., sans heurts. Il faut dire qu'au Liban, dix-huit communautés religieuses coexistent pacifiquement; mais ce pluralisme confessionnel lié à son histoire bien plus qu'à sa situation géographique, est lacéré, à cause des mains étrangères qui ont transformé ce pacifisme en bellicisme occasionnel pour leurs intérêts personnels.

Mais tout ceci ne m'empêche de la quitter à Marseille pour aller la rejoindre au Liban, dans ma ville natale et "plonger dans son sein frais » que n'égalera aucun autre sein! Voilà en quelque sorte un croisement significatif du poétique et du romanesque, du réel et du fictif chez un écrivain.

EO : Vous êtes positive dans la vie que dans votre œuvre. Vous créez du positif à partir du négatif. Votre œuvre est une véritable source de bonheur et d'inspiration pour les chercheurs, un véritable chant d'amour, de tolérance et de vie. 